Ann. Biol. anim. Bioch. Biophys., I970, 10 (4), 62I-630.

\title{
ESTIMATION DES LIPIDES CORPORELS DU POULET PAR LA MÉTHODE DE DILUTION ISOTOPIQUE DE L'EAU TRITIÉE
}

\author{
J. OUHAYOUN \\ avec la collaboration technique de Régine PENARD \\ Laboratoive de Méthodologie génétique \\ Centre de Recherches de Toulouse, 31 - Castanet-Tolosan \\ Institut national de la Recherche agronomique
}

\section{RÉSUMÉ}

La technique de dilution isotopique de l'eau tritiée, qui est adaptée ici à l'espèce Gallus gallus et appliquée à un échantillon mâle de race Wyandotte, conduit à une surestimation de I,5 p. Ioo environ du pool d'eau corporelle.

Deux méthodes de prédiction des lipides corporels $\left(\mathrm{L}_{\mathrm{E}}\right)$ du poulet sont présentées :

$$
\begin{aligned}
& \text { I. }\left\{\begin{array}{l}
\widehat{\mathrm{PCD}}=b \mathrm{E}_{\mathrm{THO}}+a \\
\mathrm{~L}_{\mathrm{E}_{1}}=\mathrm{PC}-\widehat{\mathrm{PCD}}
\end{array}\right. \\
& \text { 2. } \widehat{\mathrm{L}_{\mathrm{E}_{2}}}-\overline{\mathrm{L}}_{\mathrm{T}}=\beta_{1}\left(\mathrm{E}_{\mathrm{THO}}-\overline{\mathrm{E}}_{\mathrm{THO}}\right)+\beta_{2}(\mathrm{PC}-\overline{\mathrm{PC}})
\end{aligned}
$$

Bien que l'équation de régression linéaire du poids corporel délipidé (PCD) sur le volume de diffusion de l'eau tritiée ( $\mathrm{E}_{\mathrm{THO}}$ ) permette une estimation des lipides moins précise que l'équation de régression multiple utilisant une variable supplémentaire : le poids corporel ( $\mathrm{PC}$ ), elle offre toutefois l'avantage de se référer à un système possédant un sens biologique défini et général : la masse corporelle délipidée.

\section{I. - INTRODUCTION}

La composition corporelle instantanée d'un individu résulte de l'action des facteurs génétiques et du milieu (facteurs nutritionnels, climatiques, etc.). En particulier, la teneur en lipides corporels d'une souche animale, dans le cas où les facteurs extrinsèques sont contrôlés ou maintenus constants, est le résultat de l'expression du génotype des individus qui la composent. Les études génétiques de croissance des espèces domestiques ont généralement pour objet leur amélioration pondérale. Or, 
l'augmentation des tissus adipeux est susceptible, dans certains cas défavorables, de représenter une part importante du gain de poids ; c'est pourquoi la détermination des dépôts lipidiques au cours de la période de croissance présente un intérêt majeur.

Chez les petites espèces, la mesure directe des lipides corporels totaux par extraction est aisée ; toutefois sa lenteur limite le contrôle d'effectif́s importants et de plus cette technique a l'inconvénient de n'être réalisable que post mortem.

DELPECH et RICARD (I965) ont établi chez Gallus gallus une relation linéaire entre les dépôts adipeux viscéraux et les lipides corporels totaux. Son application à la prédiction des lipides nécessite encore l'abattage des animaux à tester et un strict contrôle du niveau énergétique alimentaire auquel ils sont soumis aut cours de leur croissance.

Plusieurs méthodes de détermination indirecte des lipides corporels in vivo ont été proposées : elles sont analysées par Dumont (I958).

Depuis les travaux de Moulton (I923), qui mettaient en évidence la constance de la teneur en eau de la masse maigre chez l'animal qui a atteint la " maturité chimique », de nombreuses études ont, de façon générale, montré que très tôt au cours de la croissance postnatale le corps délipidé atteint une composition stable. Ainsi, Pace et Rathbun (I945) ont démontré que chez le Cochon d'Inde, le Rat, le Chat et le Chien, l'eau représente 73,2 p. Ioo du poids corporel délipidé. PANARETTo (I962) indique un taux plus faible pour le Lapin : 72,6 p. Ioo $(\sigma=\mathrm{I}, \mathrm{I})$. Chez Gallus gallus, Ouhayoun (I968) trouve un résultat en accord avec ceux de PACE et RÁTHBUN : sur Ir 3 poulets d'âges compris entre 4 et I9 semaines, soumis à un jeûne total de 20 heures, l'eau représente 73,25 p. roo $(\sigma=2,099)$ du poids corporel délipidé. Cependant, alors que DEI,PECH (I966) établit une relation curviligne entre le poids corporel délipidé (PCD) (1) et l'eaut totale $\left(\mathrm{E}_{\mathrm{T}}\right): \mathrm{E}_{\mathrm{T}}=0,7 \mathrm{PCD}^{0,97}$, Ounayoun propose deux équations, dont l'une est linéaire, entre lesquelles il est statistiquement impossible de faire un choix à cause de la taille réduite de 1'échantillon d'animaux :

$$
\mathrm{E}_{\mathrm{T}}=0,736 \mathrm{PCD}-4,5 ; \mathrm{E}_{\mathrm{T}}=0,768 \mathrm{PCD}^{\mathbf{0 , 9 9 3}} \text {. }
$$

Les coefficients de corrélation entre les données de PCD et de $\mathrm{E}_{\mathrm{T}}$, naturelles et après transformation logarithmique sont, dans cette expérience, respectivement égaux à $+0,998$ I et $+0,999$ I.

L'utilisation des diverses équations proposées autorise avec des précisions différentes, la détermination du poids corporel délipidé en fonction de l'eau totale.

Les lipides totaux, représentant une charge variable sur la masse corporelle délipidée, dont ils ne modifient pas la composition, peuvent alors être aisément appréciés :

\section{Poids de lipides $=$ Poids vif $($ à jeun) - Poids corporel délipidé}

La présente note rend compte de la mise au point d'une technique de détermination du pool d'eau corporelle par mesure du volume de diffusion de l'eau tritiée et de l'établissement d'une relation entre ce volume et le poids corporel délipidé, utilisable pour l'estimation rapide des lipides chez le poulet vivant. Une seconde méthode de prédiction des lipides corporels, introduisant en plus du volume de diffusion de l'eau tritiée, une seconde variable prédictrice : le poids corporel, est discutée.

(1) DeLPECh utilise la notation PFD signifiant poids frais délipidé. 


\section{II. - MATÉRIEL, ET TECHNIQUES}

\section{A. - Détermination indivecte de l'eau corporelle totale}

\section{Méthode de dilution isotopique de l'eau tritiée.}

L'opération a porté sur 47 coquelets de race $W$ yandotte soumis à un jê̂ne total de 20 heures, âgés de 6 à I 9 semaines et de poids compris entre $8 \mathrm{I} 6 \mathrm{~g}$ et $2876 \mathrm{~g}$ ( $\overrightarrow{\mathrm{P}}=\mathrm{I} 8 \mathrm{I} 2 \mathrm{~g} ; \sigma=502,9 \mathrm{~g})$.

Le Centre d'Études Nucléaires de Saclay a fourni l'eau tritiée sous forme d'une solution isotonique ayant une activité spécifique de $5 \mathrm{mCi} / \mathrm{ml}$.

Le protocole expérimental a été le suivant :

- pesée des animaux $(\Delta \mathrm{PC}=\mathrm{I} \mathrm{g})$.

- injection intrapéritonéale d'une solution isotonique d'eau tritiée à Ioo $\mu \mathrm{Ci} / \mathrm{g}$. La dose injectée, I gramme environ par kilogramme de poids vif, a été exactement déterminée par double pesée de la seringue avant et après injection.

- trois ponctions veineuses de $\mathrm{I} \mathrm{ml}$ ont été effectuées $\mathrm{I}, 2$ et 3 heures après injection. Sur 3 individus, la cinétique d'équilibration de la radioactivité dans le plasma a été suivie par prélèvement sanguin 5, 10, 20, 30 minutes, I, 2, 3 heures après marquage.

- les échantillons de sang ont été recueillis dans des tubes en verre de 2,5 ml héparinés (héparine Choay lyophilisée), à rodage normalisé, adaptés après congélation oblique sur les six postes d'une chaîne de microdistillation sous vide. La lyophilisation du sang total a été poursuivie pendant I heure environ.

- o,50 $\mathrm{ml}$ de chacun des distillats piégés dans des microrécepteurs réfrigérés a ensuite été prélevé à la pipette et introduit dans un flacon contenant le mélange scintillant ( ${ }^{1}$ ).

- les solutions d'étalonnage ont été préparées par dilution de $p$ grammes de la solution injectable dans roo $\mathrm{ml}$ d'eau bidistillée. Dix prises d'essai de $0,50 \mathrm{ml}$ de solution diluée ont fait l'objet d'un comptage.

- la radioactivité de tous les échantillons a été comptée dans un spectromètre à scintillation Tricarb Packard, dans des flacons Nuclear-Chicago 3323 en verre de $20 \mathrm{ml}$. En utilisant I $5 \mathrm{ml}$ de solution scintillante pour $0,50 \mathrm{ml}$ d'eau marquée, avec un réglage des seuils à 50 et $I$ ooo volts et une amplification de $70 \mathrm{p}$. Ioo, l'efficacité du comptage a été de I6 p. Ioo et le bruit de fond mesuré avec $0,5 \circ \mathrm{ml}$ d'eau bidistillée a toujours été inférieur à $4^{\circ} \mathrm{c} . \mathrm{p} . \mathrm{m}$. En limitant le comptage à 300 ooo coups par échantillon, la précision statistique a été voisine de 0,004 .

Le volume de diffusion de l'eau tritiée (I'THo) a été calculé selon la formule :

$$
\mathrm{E}_{\mathbf{T H O}}=\frac{\text { radioactivité injectée (c.p.m.) }}{\text { radioactivité eau sanguine distillée (c.p.m. } / \mathrm{ml})}=\frac{\frac{a_{0} \times 100}{p} \times \mathrm{V}}{a_{i}}
$$

où $a_{\mathrm{o}}$ est l'activité (c.p.m.) de $o, 50 \mathrm{ml}$ de solution d'étalonnage obtenue par dilution de $p$ grammes de solution stock injectable dans roo $\mathrm{ml}$ d'eau bidistillée, $V$ le poids de solution stock injecté et $a_{i}$ l'activité (c.p.m.) de o,50 ml d'eau sanguine distillée.

\section{B. - Détermination divecte de l'eau corporelle totale}

\section{Méthode de dessiccation.}

Après détermination du volume de diffusion de l'eau tritiée de leur organisme, les animaux ont été sacrifiés par injection intra-veineuse rapide de $2 \mathrm{ml}$ de Nembutal, puis réfrigérés.

Les cadavres entiers non plumés ont été broyés dans un hachoir Robot-Coupe de type RCV $2 \mathrm{~A}$.

(1) Composition de la solution scintillante :

- 2.5 diphényloxazole (PPO) Amersham/Searle

- I,4 bis 2-(5-phényloxazolyl) benzène (POPOP) Nuclear-Chicago

- Naphtalène 6200 Merck

- Dioxane P. A. Merck

- Éther monoéthylique de l'éthylène glycol LAB Merck

- Xylène P. A. Prolabo 
L'eau totale a été déduite des pertes de masse dues aux trois opérations suivantes :

- broyage,

- lyophilisation d'une partie aliquote du broyat (300 g),

- étuvage à $105^{\circ}$ pendant 24 heures de $50 \mathrm{~g}$ de broyat lyophilisé.

\section{C. - Extraction des lipides du broyat lyophilisé. \\ Détermination du poids corporel délipidé}

Une variante de la technique d'extraction des lipides de DeLPEch et al. (Ig66) a été utilisée. Elle est décrite dans une note séparée (Ounayoun, 1970).

Le poids corporel délipidé des 47 animaux de cette expérience a été obtenu par soustraction du poids vif à jeun de la quantité de lipides calculée à partir de quatre extractions effectuées sur $4 \mathrm{~g}$ environ de broyat lyophilisé.

\section{III. - RÉSULTATS}

\section{A. - Estimation de l'eau totale par la méthode de dilution isotopique de l'eau tritiée}

\section{Cinétique de diffusion de l'eau tritiée dans l'organisme.}

L'examen des tableaux I et 2 montre que l'eau tritiée injectée par voie intrapéritonéale diffuse très rapidement dans l'organisme et que la radioactivité spécifique du sang atteint une valeur constante, aux erreurs aléatoires de comptage près, une heure après marquage. En effet, l'analyse de variance des mesures effectuées I, 2 ou 3 heures après injection du traceur sur 34 animaux conduit à une valeur non significative de $\mathrm{F}: 0,0027$.

Dans la suite de ce travail, le volume de diffusion de l'eau tritiée est considéré comme constant entre les temps $(t+\mathbf{~ h})$ et $(t+3 \mathrm{~h})$ et égal à la moyenne des estimations faites $I, 2$ et 3 hetres après injection de la dose d'eau tritiée.

2. Relation entre l'eau totale déterminée par dessiccation et l'eau totale estimée par dilution isotopique.

Les paramètres statistiques des séries de mesures de volume de diffusion de l'eau tritiée in vivo ( $\mathrm{E}_{\text {тн }}$ ) sont comparés dans le tableau 3 à ceux qui concernent la dessiccation des broyats corporels $\left(\mathrm{E}_{\mathrm{T}}\right)$, méthode de référence généralement adoptée.

L'équation de régression de $\mathrm{E}_{\boldsymbol{T}}$ sur $\mathrm{E}_{\mathrm{тно}}$, exprimés en grammes ( ${ }^{1}$ ), est la suivante:

$$
\widehat{\mathrm{E}}_{\mathbf{T}}=0,9975 \mathrm{E}_{\text {тно }}-\mathrm{I6,2} \text { (fig. I) }
$$

Le coefficient de corrélation est statistiquement significatif et voisin de 1'unité $(r=0,9927)$.

\section{B. - Prédiction du poids corporel délipidé}

La régression linéaire du poids corporel délipidé sur le volume de diffusion de l'eau tritiée, exprimés en grammes, a pour équation :

$$
\widehat{\mathrm{PCD}}=\mathrm{I}, 3 \mathrm{I} 32 \text { Е тно }+ \text { 30,I (fig. 2) }
$$

(1) La densité de l'eau à la température ambiante ( $\left.\mathrm{I}^{\circ}\right)$ étant très voisine de l'unité $\left(0,99^{8} 62\right)$, les poids et les volumes ont été confondus dans tous les calculs. 


\section{TABLEAU I}

Évolution de la radioactivité spécifique du sang veineux, exprimée en pourcentage de la radioactivité spécifique mesurée 3 heures après injection intrapéritonéale d'eau tritiée

\begin{tabular}{|c|c|c|c|c|c|c|c|c|c|}
\hline \multirow{2}{*}{ No } & \multirow{2}{*}{ animal } & \multirow{2}{*}{$\begin{array}{l}\text { Poids vif } \\
\text { à jeun }(g)\end{array}$} & \multicolumn{7}{|c|}{ Radioactivité du sang veineux } \\
\hline & & & $5 \mathrm{mn}$ & $10 \mathrm{mn}$ & $20 \mathrm{mn}$ & $30 \mathrm{mn}$ & $1 \mathrm{~h}$ & $2 \mathrm{~h}$ & $3 \mathrm{~h}$ \\
\hline & 1 & 1427 & 73,01 & 104,63 & 101,17 & 99,93 & 100,61 & 100,18 & 100 \\
\hline & 2 & 1391 & 84,57 & 106,48 & 104,32 & 100,63 & 99,37 & 100,07 & 100 \\
\hline & 3 & 892 & 63,18 & 89,71 & 98,77 & 100,80 & $100,2 ' t$ & 99,67 & 100 \\
\hline
\end{tabular}

TABLEAU 2

Volume moyen de diffusion de l'eau tritiée de 34 mâles, 1,2 et 3 heures après injection du traceur

\begin{tabular}{|c|c|c|c|c|}
\hline Prélèvements & $\begin{array}{l}\text { Volume moyen } \\
\qquad(\mathrm{ml})\end{array}$ & $\begin{array}{c}\text { Écart-type } \\
(\mathrm{ml})\end{array}$ & $F^{(1)}$ & $\frac{\text { Volume moyen }}{\text { Poids vif } \times 10^{-2}}$ \\
\hline $\begin{array}{ll}1 & h \\
2 & h \\
3 & h\end{array}$ & $\begin{array}{l}1163,4 \\
1169,4 \\
1165,4\end{array}$ & $\begin{array}{l}334,62 \\
335,01 \\
331,06\end{array}$ & 0,0027 N.S. & $\begin{array}{l}69,67 \\
70,03 \\
69,80\end{array}$ \\
\hline
\end{tabular}

(1) N.S. : Test non significatif $(\mathrm{P}>0,05)$.

TABIEAU 3

Expression de $E_{\mathbf{T}}$ et de $E_{\mathbf{T H o}}$ par rapport au poids vif et au poids corporel délipidé de 47 coquelets

\begin{tabular}{|c|c|c|c|c|}
\hline \multirow{3}{*}{ Méthode } & \multicolumn{4}{|c|}{ Eau totale } \\
\hline & \multicolumn{2}{|c|}{$\%$ Poids vif } & \multicolumn{2}{|c|}{$\%$ PCD } \\
\hline & $m$ & $\sigma$ & $m$ & $\sigma$ \\
\hline $\begin{array}{l}\text { Dessiccation....... } \\
\text { Dilution THO } \ldots \ldots\end{array}$ & $\begin{array}{l}68,66 \\
69,87\end{array}$ & $\begin{array}{l}2,657 \\
2,730\end{array}$ & $\begin{array}{l}73,49 \\
74,80\end{array}$ & $\begin{array}{l}2,083 \\
2,139\end{array}$ \\
\hline$t \ldots \ldots \ldots \ldots \ldots \ldots$ & & & & \\
\hline
\end{tabular}

* Écart significatif $(0,01<\mathrm{P}<0,05)$.

** Écart hautement significatif $(\mathrm{P}<0,01)$. 


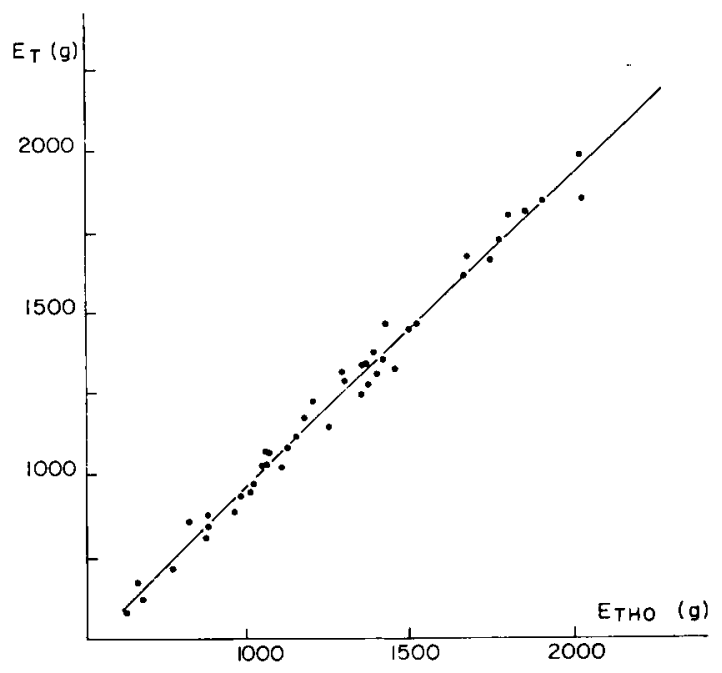

FIG. I. - Droite de régression de l'eau totale $\left(\mathrm{E}_{\mathrm{T}}\right)$ sur le volume de diffusion de l'eau tritiée ( $\mathrm{E}_{\mathrm{TH}}$ )

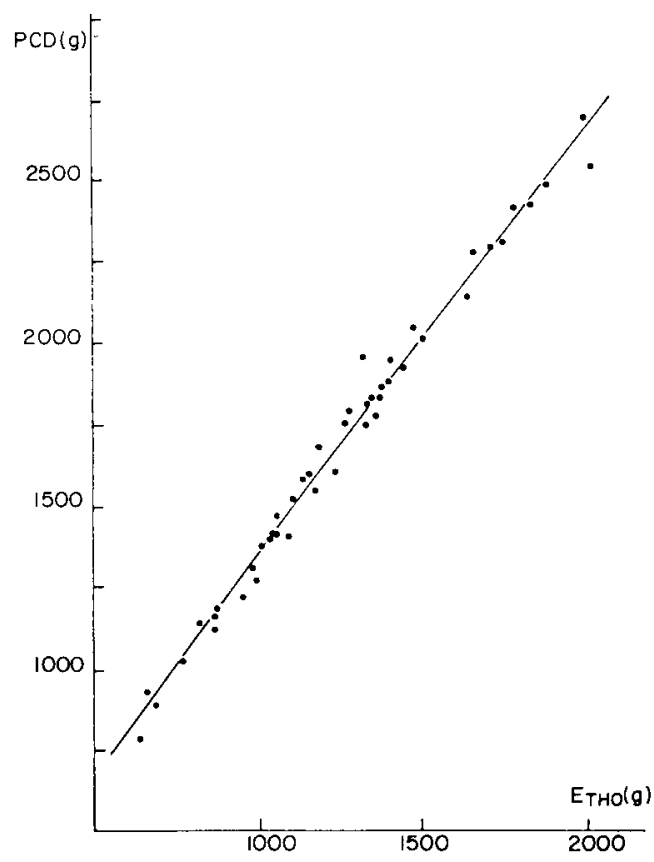

FIG. 2. - Droite de régression du poids corporel delipide (PCD) sur le volume de diffusion de l'eau tritiée ( $\left.\mathrm{E}_{\mathrm{TH}} \mathrm{O}\right)$ 
La corrélation entre les couples de données est étroite $(r=+0,9946)$. L'erreur standard sur la prédiction du poids corporel délipidé : $\mathrm{ES}= \pm \sigma \mathrm{PCD} \sqrt{\mathrm{I}-r^{2}}$ est égale à 48 ,o g pour la valeur expérimentale moyenne de la variable prédictrice Етно.

\section{C. - Estimation des lipides totaux $\left(\mathrm{L}_{\mathrm{E}}\right)$}

I a variabilité des valeurs des lipides totaux dans l'échantillon analysé est très grande. DELPECH et RICARD (I965) ont fait la même constatation sur des lots d'oiseaux pourtant soumis à des conditions trophiques et d'ambiance contrôlées. Dans le cas présent, le poids moyen des lipides totaux extraits, exprimé en pourcentage du poids vif et de $6,59(\sigma=2,084)$.

I. $L_{E_{1}}=P C-\widehat{P C D}$.

La corrélation entre les lipides totaux extraits $\left(\mathrm{L}_{\mathrm{T}}\right)$ et estimés $\left(\mathrm{L}_{\mathrm{E}_{1}}=\mathrm{PC}-\mathrm{PCD}\right)$ exprimés en grammes, est significativement différente de zéro $(r=+0,7738)$.

\section{Prédiction directe des lipides totaux.}

Les lipides corporels ont été également prédits en introduisant dans une équation de régression multiple deux variables prédictrices : le volume de diffusion de l'eau tritiée et le poids corporel, qui sont chacune en corrélation assez étroite avec les lipides totaux extraits :

$$
\begin{aligned}
& r \mathrm{E}_{\text {тн }}, \mathrm{L}_{\mathrm{T}}=+0,7450 \\
& r{\mathrm{PC}, \mathrm{L}_{\mathrm{T}}}=+0,7969
\end{aligned}
$$

La forme de cette équation est la suivante :

$$
{\widehat{\mathrm{L}_{2}}}_{\mathrm{T}}-\overline{\mathrm{L}}_{\mathrm{T}}=\beta_{1}\left(\mathrm{E}_{\mathrm{THO}}-\overline{\mathrm{E}}_{\mathrm{THO}}\right)+\beta_{2}(\mathrm{PC}-\overline{\mathrm{PC}})
$$

Ses paramètres ont pour valeur :

$$
\begin{aligned}
& \beta_{1}=-\quad 0,41693 \\
& \underline{\beta}_{2}=-0,37 \mathrm{I} 02 \\
& \overline{\mathrm{I}}_{\mathrm{T}}=\mathrm{I} 23,02 \\
& \overline{\mathrm{E}}_{\text {тно }}=\text { I } 263,23 \\
& \overline{\mathrm{PC}}=\mathrm{I} 8 \mathrm{I} 2,00
\end{aligned}
$$

Le coefficient de corrélation multiple $\mathrm{R}^{2} \mathrm{~L}_{\mathrm{T}}, \mathrm{E}_{\mathrm{TH}}$, PC est significativement différent de zéro : $+0,76$ I 0 .

L'erreur standard sur la prédiction des lipides :

$\mathrm{ES}= \pm \sigma \mathrm{L}_{\mathrm{T}} \sqrt{\mathrm{I}-\mathrm{R}^{2}}$ est égale à $25,6 \mathrm{~g}$.

La corrélation entre les lipides totaux extraits $\left(\mathrm{L}_{\mathbf{T}}\right)$ et prédits $\left(\mathrm{L}_{\mathrm{E}_{2}}\right)$ est étroite : $r=+0,87$ × 3 .

\section{IV. - DISCUSSION ET CONCLUSION}

Les méthodes indirectes d'analyse corporelle in vivo et surtout celles qui sont basées sur le principe de la dilution isotopique ont considérablement facilité l'étude descriptive des espèces animales. Outre l'intérêt de leur grande sensibilité et de leur 
maniement simple, leur application a l'avantage de pouvoir être répétée sur un même individu et donc de permettre des investigations dynamiques sur sa composition corporelle. La dilution de l'eau tritiée en particulier autorise l'estimation du pool d'eau corporelle de toutes les espèces et notamment de celles dont la taille exclut l'application de la méthode de dessiccation.

Chez Gallus gallus, la radioactivité spécifique du sang veineux, compartiment d'exploration de l'ensemble de l'espace virtuel de diffusion de l'eau tritiée semble constante de I à 3 heures après injection intrapéritonéale du traceur (tabl. 2). La méthode d'extrapolation de CACHERA et BARBIER (I945) dont 1'application est indispensable en cas de renouvellement rapide du composant corporel étudié n'a donc pas été utilisée ici. Pour autant que la dessiccation puisse être considérée comme une référence fiable, la méthode de dilution de l'eau tritiée conduit à une surestimation de l'eau corporelle (tabl. 3). Cet excès représenterait le pool d'hydrogène échangeable envisagé par KROG et Ussivg (I936) qui, d'après les données rapportées par Panaretto (I963), varierait de 0,5 à $3 \mathrm{p}$. Ioo du poids vif selon les espèces. Les échanges entre l'isotope de l'hydrogène utilisé comme traceur et les atomes labiles d'hydrogène de divers composés organiques biologiques conduisent ainsi à envisager dans le cas présent un volume de diffusion de l'eau tritiée supérieur de I,5 p. roo à celui occupé par l'eau corporelle totale.

Les deux méthodes d'estimation in vivo des lipides présentées n'ont pas la même portée pratique.

La première, qui utilise un système de référence présentant de faibles variations de composition inter-espèces : la masse corporelle délipidée, confère aux résultats un sens physiologique précis. La charge lipidique du corps, immédiatement accessible, est égale à la différence entre le poids corporel et une fonction simple du volume de diffusion de l'eau tritiée.

Cependant, les valeurs relativement élevées ( 2 p. Ioo) du coefficient de variation du volume de diffusion de l'eau tritiée, exprimé par rapport au poids corporel délipidé, d'une part, et de l'erreur standard sur la prédiction du poids corporel délipidé, donc sur l'estimation des lipides ( $\mathbf{S} S= \pm 48,0 \mathrm{~g}$ ), d'autre part, montrent que si cette méthode est applicable à la comparaison de souches variées et saines sans qu'il soit nécessaire d'établir préalablement pour chacune d'elles une nouvelle fonction $\widehat{\mathrm{PCD}}=f\left(\mathrm{E}_{\mathbf{T} \mathrm{Ho}}\right)$, elle ne l'est pas avec une précision suffisante à la caractérisation d'individus isolés.

I a seconde méthode fait appel à deux variables prédictrices, chacune en corrélation assez étroite avec les lipides totaux : le poids corporel et le volume de diffusion de l'eau tritiée. La valeur de l'erreur standard sur la prédiction des lipides est sensiblement inférieure à celle qui est attachée à la précédente estimation $(25,6 \mathrm{~g})$.

Toutefois, l'équation de régression multiple proposée ne présente d'intérêt que pour la détermination des lipides corporels des coquelets appartenant à la souche animale utilisée pour son établissement : aucun argument ne permet en effet de préjuger des relations susceptibles d'exister entre chacune des deux variables prédictrices retenues et les lipides corporels d'une autre souche. Ia comparaison de types génétiques ou de régimes nutritionnels, quant à leur action sur le stockage des lipides corporels, suppose donc que soient préalablement établies des équations de régression multiple de la forme présentée en nombre égal à celui des effets à tester.

En conclusion, bien que la méthode basée sur le système de référence de la masse 
corporelle délipidée conduise à des résultats moins précis que celle qui introduit deux variables prédictrices, elle présente l'avantage notable d'avoir une portée plus générale. Elle semble propre à faciliter chez Gallus gallus l'étude de l'action des facteurs génétiques et trophiques sur la teneur du corps en lipides notamment.

\section{Reçu pour publication en mai $19 \% 0$.}

\section{SUMMARY}

\section{ESTIMATION OF BODY LIPIDS IN CHICKENS BY THE ISOTOPE DILUTION MFTHOD WITH TRITIATED WA'TER}

Tritiated water space in 47 starved cockerels of different ages was compared with bodywater pool estimated by drying the complete ground carcass.

Compared with drying, the reference method, isotope dilution overestimated bodywater by I.5 p. Ioo, and suggests for the species Gallus gallus a pool of exchangeable water representing $69.87 \mathrm{p}$. Ioo of bodyweight and $74.80 \mathrm{p}$. Ioo of fat-free bodyweight (table 3 ).

A linear relation was shown between the tritiated water space (E $E_{\text {rno }}$ ) and fat-free bodyweight (PCD). The equation was : $\widehat{\mathrm{PCD}}=\mathrm{I} .3 \mathrm{I} 3$ 2E. THo + 30.I (fig 2). There was a very close correlation between the pairs of values $(r=+0.9946)$. Its application allows rapid prediction of total lipids in live chickens by weighing and measurement of tritiated water space.

The correlation between total lipids of the 47 cockerels, estimated by chemical extraction $\left(\mathrm{L}_{\mathrm{T}}\right)$, and total lipids estimated from the above relation $\left(\mathrm{L}_{\mathrm{E}_{1}}\right)$, was very close $(r=+0.7738)$

A second method for estimating lipids in chickens is presented. It consists of the introduction of two prediction variables, bodyweight (PC) and tritiated water space, into a multiple regr $\epsilon s i o n$ equation : $\widehat{\mathrm{L}}_{\mathrm{E}_{2}}-\overline{\mathrm{L}}_{\mathrm{T}}=\beta_{1}\left(\mathrm{E}_{\mathrm{THO}}-\overline{\mathrm{E}}_{\mathrm{THO}}\right)+\beta_{2}(\mathrm{PC}-\overline{\mathrm{PC}})$.

The value for the multiple correlation coefficient was significantly different from zero $\left(\mathrm{R}_{\mathrm{LT}}{ }_{\mathrm{T}} \mathrm{E}_{\mathrm{THO}}, \mathrm{PC}=+0.76 \mathrm{I} \mathrm{o}\right)$. The correlation between extracted lipids ( $\left.\mathrm{L}_{\mathrm{T}}\right)$ and predicted lipids $\left(\mathrm{L}_{\mathrm{K}_{2}}\right)$ was close $(r=+0.87$ I 3$)$.

Although increasing the precision of prediction of body lipids, the relation in which the fatfree bodyweight does not appear, as it does in the first system, is really applicable only to the strain of animal for which it has been established. In fact it is impossible to predict particularly the values for the partial $\mathrm{rcgression}$ coefficients $\beta_{1}$ and $\beta_{2}$ in other animal populations.

\section{RÉFÉRENCES BIBLIOGRAPHIQUES}

Cachera R., Barbier P., i94I. L'épreuve du rhodanate de sodium, méthode de mesure du volume des liquides interstitiels. C. R. Soc. Biol., 135, rr75.

Delpech P., Ricard F.-H., I965. Relation entre les dépôts adipeux viscéraux et les lipides corporels chez le Poulet. Ann. Zootechn., 14, I8I-I89.

Deт.PECH P., I966. Le poids frais délipidé chez Gallus gallus L, Relations qui unissent ses constituants. C. R. Acad. Sci. Paris. 263, I735-1738.

Defpech P., Guezel M., Leclerco B., I966. Méthode d'extraction des lipides en continu et à chaud par le mélange azéotrope benzène-éthanol-eau. Rev. franç. Corps gras, 10, $6 \mathrm{p}$.

Dumont B. L., I958. Méthodes indirectes de mesure de la graisse corporelle des mammifères. Les Cahiers techniques du Centre national de la Recherche scientifique, Paris, I58 p.

Krogh A., Ussing H. H., 1936. The exchange of hydrogen between the free water and the organic substances in the living organism. Skand. Arch. Physiol., 75, 9o-I04.

Moulton C. R., r923. Age and chemical development in mammals. J. Biol. Chem. 5\%, 79.

Ouhayoun J., I968. Résultats non publiés. 
OUhayoun J., I970. En préparation.

Pace N., Rathbun E. N., I945. Studies on body composition. 1II. The body water and chemically combined nitrogen in relation to fat content. J. Biol. Chem., 158, 685 .

Panaretto B. A., Ig62. Body composition in vivo. I. The estimation of total body water with antipyrine and the relationship of total body water to total body fat in rabbits. Aust. J. Agric. Res., 14, 594.

Panaretro B. A., Till A. R., x963. Body composition in vivo. II. The composition of mature goats and its relationship to the antipyrine, tritiated water, and $\mathrm{N}$-acetyl-4-Aminoantipyrine spaces. Aust. J. Agric. Res., 14, 926-943. 